\title{
How do you exercise with epilepsy? Insights into the barriers and adaptations to successfully exercise with epilepsy
}

\begin{abstract}
Exercise has been shown to be a physiological and psychological benefit for people with epilepsy (PWE). However, barriers prevent many PWE from exercising safely and confidently. This research explored current perceived barriers to exercise and adaptation techniques used by PWE in order to maintain physical activity levels. Three focus groups (23 participants per group) and three semi-structured interviews were conducted (11 participants total). Constructive grounded theory was used to frame the study and analyse the findings, presenting new insight into the motivation, perceived barriers, and adaptation techniques used to exercise. The main motivator to maintain physical activity levels was the benefit of exercise on their physical and mental health. This was shown in an increase in mood, higher social interaction, and perceived improvement in overall physical health as a result of exercise. Current barriers to exercise included a fear of injury, lack of social support, and exercise-induced seizures (e.g., through overheating and/or high exercise intensity level). Adaptation techniques used were self-monitoring through the use of technology, reducing exercise frequency and intensity level, and exercising at certain times of the day. The importance of social support was shown to provide increased confidence and positive encouragement to exercise, contrasting with family and friends worrying for his/her safety and medical professionals requesting termination of some physical activities. These findings provide new insight into current adaptation techniques that are used and developed by PWE to overcome common barriers to exercise. These new additions to the literature can lead to further development of such techniques as well as examine current medical professionals' knowledge of the benefits of exercise for PWE.
\end{abstract}

\subsection{Introduction}

For people with epilepsy (PWE), exercise has shown to be beneficial for seizure control, decreases the side effects associated with medication, and improves overall wellbeing [1-3]. Furthermore, research investigating quality of life (QoL) shows the improvement in mood and QoL for PWE after a 12 week exercise program [3]. Recent qualitative research has confirmed these benefits of exercise, with participants voicing that exercise increases their physical health, decreases stress levels, increases self-esteem, positively impacts mood, and improves QoL overall [4, 5]. Although both qualitative and quantitative research have presented the positive impact of sports and exercise for a person with epilepsy [1-3, 5, 6], the prevalence of regular exercise for a person with epilepsy is 
lower compared to the general public [7], as there are still common barriers (both physical and psychosocial) that prevent many PWE from feeling the benefits of exercise $[4,5,8]$. The main barriers to sports and exercise reported are fear of the seizure occurring [4, 8], fear of seizure-related injuries [9], stigma [5, 10] and incorrect advice from medical professionals [4, $5,11,12,13]$. As a result, this inactivity has shown to cause social isolation, low self-esteem, weight gain, and depression $[5,9,11]$.

Although the benefits of and barriers to sports and exercise have been identified, there is a lack of literature on the coping mechanisms and adaptation techniques used by PWE to exercise safely and confidently. This research was an exploratory study investigating possible barriers and coping strategies of exercising with epilepsy. Our aim was to use qualitative methods to explore ways of enhancing physical activity levels for PWE by examining the barriers to exercise, current adaptation techniques used to overcome these barriers, and common exercise activities and intensity levels.

\subsection{Methods}

\subsection{Participants}

Prior to recruitment, ethical approval was gained from Bournemouth University. Participants were initially recruited via Epilepsy Action's website, newsletter, and support groups around the South West of England. The recruitment area was extended to any region of England as a result of limited participants able to take part within the South West.

Participant inclusion criteria were that each had a medical diagnosis of epilepsy; no comorbid physical condition that could prevent exercise; at least 18 years of age or older; and live within two hours travel distance to Bournemouth. After the first two focus groups, the ability to travel was disregarded for those willing to take part in online focus groups or interviews. 
withdrawal prior to participating in a focus group or interview, the total number of demographic data. This accounts for individuals having more than one seizure type.

\begin{tabular}{|l|l|}
\hline Gender & F Females; 4 Males \\
\hline Age & Mean: 42 years \\
& Range: $18-60$ years \\
\hline Seizure type & $\begin{array}{l}\text { Tonic-Clonic: } 11 \\
\text { Partial seizure: } 3 \\
\text { Absence: } 2 \\
\text { Myoclonic: } 1\end{array}$ \\
Mean time since diagnosis & $\begin{array}{l}\text { 18 years } \\
\text { Range: } 8-49 \text { years }\end{array}$ \\
& White- British: 10 \\
\hline Ethnicity & Black-British: 1 \\
\hline
\end{tabular}

65

Frequency and type of exercise activity varied amongst the participants. For each participant, exercise frequency depended on seizure frequency, e.g., the greater the frequency of seizures, the fewer times he/she exercised. In a week without seizures, exercise frequency ranged from 2- 10 times per week, depending on the participant and his/her level of seizure control. For example, one participant (tonic-clonic seizures; seizure frequency varying from 1-2 a month to 1 every couple of months) exercised (rowing, running, strength training) at least 6-10 times a week. However, in a week that she had a tonic-clonic seizure, this would be five times or less. The average exercise frequency was 4 times per week. Walking, running, 
swimming, cycling, rowing, spinning, strength training, and squash were the activities undertaken. The most common activities were walking, running, and swimming.

2.2 Focus groups and semi-structured interviews

Focus groups and individual semi-structured interviews were used to explore the exercise experiences of PWE. Focus groups were chosen to 'provide a forum where participants feel more comfortable discussing sensitive issues' [14, p. 30]. Through discussing their experiences with others who have the same condition, it was intended that the participants may feel more at ease in discussing potentially sensitive topics [15]. To prevent an individual from dominating the conversation, the main researcher (SC) provided 'active people management' [14, p. 31] in order to encourage quieter participants to contribute. The first two focus groups were conducted in Bournemouth University interview rooms and the third focus group was conducted online using videoconferencing technology. Each focus group lasted $1 \frac{1 / 2}{2}$ to 2 hours. Semi-structured interviews were conducted as a result of travel limitations and low recruitment numbers. Semi-structured interviews were scheduled at a time suitable for each participant and were conducted online using videoconferencing technology. Interviews lasted 1 to $1 \frac{1 / 2}{2}$ hours each. The focus groups and interviews were audio-recorded after written and verbal consent from the participants. Pseudonyms are used to protect the participants' confidentiality.

Questions were asked around the common themes of barriers to exercise, adaptation methods, and benefits of exercise. Further, the focus groups and interviews permitted an open discussion of themes (e.g., related to exercise adherence, social support, etc.) that may be currently absent from research. A topic guide was used in the focus groups and interviews in order to explore these themes.

Sample questions asked: 
1) Tell me about your experience exercising with epilepsy.

2) What type of exercise do you do?

3) How often do you exercise?

4) What motivates you to exercise?

5) Are there any barriers to exercise?

6) How do you overcome these barriers?

7) Have you discussed exercise with your doctor? If so, what does he/she say?

8) How do your family/friends feel in regard to your exercise routine?

\subsubsection{Trust and rapport}

Prior to starting each focus group or interview, trust and rapport were built with the main researcher and participants through conversations on the phone, email, and in person/online. This was key in developing comfort in the research setting and to ease any of the participants' concerns prior to the start of the sessions.

\subsection{Constructionist grounded theory}

The focus groups and interviews were analysed using constructionist grounded theory (CGT). As CGT recognizes the impact of the researcher upon the research, this methodology was chosen as it aims to 'give a voice to the subject' [16, p.11]. Data were transcribed and analyzed using CGT in order to develop possible models that could be explored within future research. The analysis process was conducted as follows [17]: Initial coding began after the first focus group. This involved verbatim transcription followed by coding to discover the important themes that emerged. This process then led to supplementary questions for the next focus group. Following this, focused coding was conducted through refining the categories and through the use of further data collection, establishing the categories and their connections further. Next, theory development occurred. This process laid the foundations of the developing theory, leading to further interviews and refinement of the theory over time. 
123 Lastly, themes that emerged from the last focus group and interviews were then used in

124 discussions with the earlier participants to develop the theory further. This last step involved one to one discussions with six earlier participants (via phone and/or email).

This analytical process was conducted until no new topics were discovered with this participant group and the emerging theory allowed a depiction of the barriers, benefits, and adaptation methods used by PWE to exercise. Following analysis, member checking [18] was conducted through presenting these findings back to the participants in order to assess if the participants felt that they could recognize their experiences in the findings.

\subsection{Reflection}

As the main researcher has epilepsy, this was discussed with the participants prior to the start of the focus groups and interviews in the case of a seizure occurring. To limit researcher bias, reflection techniques were followed throughout the research process. Such techniques included discussions with the co-author, self-reflection, and memo writing $[4,5]$.

These reflection methods occurred prior to and after interviews and focus groups, during transcription, as well as throughout the analysis process. Reflection allowed for further development of the analysis through memo writing, allowing connections between themes, and further solidification of the grounded theory method. Great care was taken so that the researcher's experiences did not dominate the interviews or analysis. Participants remarked that due to the main researcher having epilepsy as well, they felt an atmosphere of comfort and openness to discuss topics they had not discussed with others before [5].

\subsection{Results}

Results revealed core categories of motivation to exercise, barriers to exercise, adaptation methods, and the impact of social support. Within these core categories, 
connections between themes emerged, providing a continuously cyclical and evolving portrayal of the impact of epilepsy on PWE's exercise routine [See Fig. 1].

\subsection{Motivation to exercise}

For the participants, there were many different motivating factors in deciding to exercise as well as maintaining an exercise routine. The overall feeling of the benefit of exercise to their physical and mental health was commonly discussed.

(Rebecca, Interview): I love it, I love exercise. I love it. I love, when I get on the treadmill or I run home or I go for a run. I don't have an issue, I don't have a mental block at all! Like a lot of people hate exercise, I love it. When I put my shoes on and go for a run, there's nothing better. ... exercise for me is very, very relaxing.

(Dylan, Focus group): Um... I've always loved to exercise cause it's a part of my life. Um, I enjoy it, keep a healthy lifestyle cause they say if you exercise you keep healthy and fit. And, also it's a good social with my mates and family as well. It gets everyone together.

Partaking in exercise made the participants feel physically healthy (e.g., decreased weight

gain as a result of seizure medications), happier, relieved stress, and increased social

interaction. Through partaking in exercise, participants felt that they were not allowing

epilepsy to take over their life.

(Heather, Focus group): I always, personally, just get on with it. My attitude towards my epilepsy is, I mean I was diagnosed with it at age 14. So I've had it most of my life now when I work out. So I've always taken the approach that epilepsy doesn't own me. I never ever consciously think about it. I'm fortunate that mine's quite controlled.

Not allowing their epilepsy to constantly prevent them from exercising, some participants felt that consistent exercise improved seizure recovery.

(Kimberly, Interview): I tend to find the fitter I am, the healthier I am, the shorter the recovery time [from a seizure] as well... I ... I don't know, I know I feel better after seizures. Like I've been able to just get up and about the same day whereas it [seizure] used to, just knock me out for a couple of days. ... But actually since I've been better health wise, it's [recovery from a seizure] actually not been too bad. 
In addition to the benefit of exercise aiding seizure recovery, participants shared the positive impact of exercise upon stress levels and overall health. Subsequently, they saw a decrease in their seizure frequency. Although the benefits of exercise and importance of physical activity within their lives were discussed, the barriers to exercise were also shown to be prevalent for many of the participants.

\subsection{Barriers to exercise}

Throughout each of the focus groups and interviews, there was a common theme of barriers that prevented exercise. Although reasons typically seen, i.e., not enough time, family commitments, etc., were discussed, the majority of reasons for those with uncontrolled seizures were epilepsy-related. First, a common worry was the impact of high intensity exercise on triggering a seizure and knowing his/her limits.

(Kimberly, Interview): The main thing that comes up really is like, the safety aspects of it. Just making sure you're safe more than perhaps... you'd worry about it if you didn't. ... Um,.. That's probably the main thing for me. Perhaps not training to or not pushing to the same intensity all the time that other people can. Cause it's always that thing in the back of your mind of, 'mmm, am I going to push a little bit too hard and have a seizure?'

For some participants, they directly saw a link to increased exercise intensity and increased seizure activity.

(Vanessa, Focus group): I continued to have a few more seizures all while exercising on the cross trainer or running. That was over a couple of years, umm, and it all seemed to be when I was doing very fast, active, high heart rate. So, any sprint work, up hills, that would be when it [a seizure] happened.

(Dylan, Focus group): I always have a fit [seizure] if I'm playing, doing exercise. It is a certain kind of exercise, see, more endurance exercise, long distance. So, when playing football for example, cause I always seem to have a fit [seizure] playing football. No matter what. I'm pretty playful on the pitch, I start running and then after $15 \mathrm{~min}$, I'll go into a seizure. It always happens and I've never understood why. 
Not only was a direct link to exercise-related seizures discussed, but the concern of having no one to exercise with also increased the likelihood of staying at home rather than partaking in exercise or sports.

(Penelope, Focus group): My only problem is, um, I won't do it by myself. So any running, if my husband isn't available, friends aren't available, then I won't do it. Which makes sense.

Additionally, one more barrier to exercise was medical advice provided to the participants.

Participants reported medical professionals did not directly recommend exercise or sports for the participants' medical and/or psychological well-being. For some, the lack of advice was frustrating, but for others being told to stop exercising by neurologists, family members, and friends resulted in a negative psychological impact.

(Heather, Focus group): I’ve, I’ve got to say, I don't think I've ever had any information about exercise when I've gone to see my...my... consultant or my epilepsy nurse.

(Dylan, Focus group): From my experience, talking to my neurologist, they informed me to stop playing sports. Cause they said that, if I'm having fits [seizures] playing sports then I shouldn't play, it's as simple as that, they said to me.

Researcher: Did you say anything back to them or, how did you react to that?

Dylan: I was distraught cause sports are with me for all my life. And like I said, I almost made it to the professional level, but being told I can't play, I should stop, it kind of.. saddened me.

(Samantha, Focus group): So... my parents worry quite a lot. I like cycling, umm... but, yeah, I think my parents worry too much (little laugh). Umm, so I could do that [cycle], but I think they worry I'll overheat. Because I have overheated in other things before. So they'll worry I'll overheat then. And then there's no one else that can go out with me, so I have to go out by myself. So it's just safer to walk.

Lastly, medication side effects, e.g., fatigue, were obstacles which caused participants not to exercise to the amount they desired. However, exercise was also one method that helped ease their medication side effects. 
(Heather and Adam, Focus group):

Heather: Actually yeah, like I said when I moved onto Keppra, I had no get up and go... You probably couldn’t have dragged me out to go for a run... But, like I say, you come through it. But I would definitely say when the side effects start, it was so... nausea and the tiredness and it made me not want to [exercise]. But once you push through that barrier of initially feeling tired, you feel the benefit afterwards. So we were saying earlier, once you've had that high of having done a run, getting that blood moving.

Adam: yeah, yeah

Heather: feeling generally really good. You say, 'Right, I'll just make sure I push through that horrendous side effect. The first K will be a killer, more so than it normally is. But, I know I'll feel better at the end of it for doing it.'

Although there were constant barriers shared, it seems that adapting to one's epilepsy is one method of helping to increase or maintain exercise levels.

\subsection{Adaptations}

One method that participants used to maintain an exercise routine was to recognize their seizure triggers, change exercise type and intensity level according to seizure activity, and use technology. First, one common method discussed was to acknowledge his/her seizure triggers and either rest or modify his/her exercise activity.

(Kimberly, Interview): Getting over tired is [a trigger]. Um, and if I know I've not slept well or something, I think it is, I do think it is partly my responsibility not to put other people in the boat in danger. So... if I know I'm overtired, don't go and get in a boat and go three miles down the river (little laugh). So... I'll do that, just have a day off or something.

(Rebecca, Interview): Yeah, so, specifically when I'm doing any weight training on the right side. Because that does send out a very, very specific, I mean I have headaches ALL the time. But, the seizure headaches are something very, very specific and I just know, 'Oh no, this is not going to have a good outcome at all.' And so when I'm doing weight training, there comes a point where I get that feeling where I need to stop really rather quickly or I would have a seizure.

Researcher: It's a different feeling compared to just say, normal fatigue?

Rebecca: Very, very different. It feels a bit more.... normal seizures when I'm just tired, they just kind of creep up, I don't know how to explain it. It's not as.. it's not as..umm..intense in the arm and in the leg as when I'm exercising. So when I'm 
exercising I can feel, 'Oh my word, that is painful, really painful. Sharp, sharp pain.' And I will feel it building up. I have an aura of about 5 min. With each seizure I get that same sort of feeling. Which can go for quite a while. Umm, but when the aura comes I then get that nausea and that's the ticket. Whereas when I'm exercising, I would stop before I even get to the point where I have that nausea. So all the muscle pain is there, the headaches there, everything's similar, but the nausea. So I stop before that point.

A common method of preventing seizures in relation to exercise is to change one's intensity level or exercise type. This was shown to be as a result of recognizing pre-seizure symptoms as well as knowing their triggers.

(Vanessa, Focus group): But I just curb my exercise, so I don't exercise to the level where I'm happy. So I go off to boot camp and we will do sprints, and I won't sprint.

(Samantha, Focus group): Umm, I just normally do walking because if I do any of the other, more like physical activity, like playing sport games, I don't know, like cricket, I'll overheat too quickly and I'll have a seizure.

The use of technology to track seizure activity in relation to exercise was discussed as a method of gaining a sense of control of their epilepsy. One technique used by two of the participants was a heart rate monitor:

(Vanessa, Focus group): I don't feel my seizures come on, but I know when I've tracked them on my heart rate monitor and my Garmin, I know it's [heart rate] been on the decline. That the body has gone into seizure... [To set my heart rate max,] I just go by the standard guidelines, so 220 minus my age and then work within $75 \%$ of that. So in my recognising, I'm allowed to go 170 beats per minute...heartrate [sic]. And if I go beyond that, I have my little watch set up so I get an alarm that goes off. Sometimes I ignore it, cross my fingers, and more often than not I stay within that.

One participant shared the common trigger of overheating as a problem for maintaining exercise. His solution was to use a type of thermometer to help him keep under control. As a result of increased temperature, this participant also uses an ice pack to cool himself down in order to prevent a seizure. Another participant changes the time of day she exercises to reduce the chances of triggering a seizure.

(Samantha, Focus group): 
Samantha: I have to make sure that it's at the right time of day.

Researcher: So what would that be?

Samantha: So [in the summer] it can't be too near 5:00pm. I wouldn't start any physical exercise at 4:00 in the afternoon.

Researcher: You wouldn’t start it?

Samantha: No, ... I would do it in the morning. I don't really count, I don't really think that walking is like physical.... But if I was to do any physical exercise I'd probably do it in the morning.

Overcoming any barriers to exercise through adaptations was one key aspect of finding ways to not let epilepsy control the participants’ lives. These adaptation techniques were particularly useful when discussed in connection with positive or negative social support.

\subsection{Social support}

Participants reported a mixture of positive and negative aspects of friends, family, and medical professionals providing social support. Firstly, although (as shown in 3.2) medical advice was mixed in regard to recommending exercise, some participants did report that his/her neurologist did suggest that epilepsy should not be a barrier to exercise.

(Adam and Heather, Focus group):

Adam: I mean, cause last summer I should have done [a race] in France, which is this big ultra-run which goes around the Alps and cause I had the hamstring injury, I... Before I actually put my entry into it, I actually got, consulted the epi specialist, ... And basically they were like, 'Why, why, why are you asking me?' Like,

Heather: You don’t need to... yeah

Adam: 'Don't talk about it, just go do it.' I mean, I don't know if it's because of his army background and he's like, 'Well, don't be such a wimp' or... (laughs)

Adam: ... But yeah, effectively he said, 'There’s no reason why you shouldn’t be doing it.'

Furthermore, some participants also expressed that they felt unable to discuss the topic of exercise with his/her neurologist because of time restraints. Consequently, any concerns on the topic of exercise were not readily discussed with his/her neurologist. 
increased sense of security when exercising with others.

(Denise, Interview):

Researcher: What makes you feel safe when exercising?

Denise: When there's people around me.

Friends and family do worry for the participants. However, participants reported friends and

family realizing the importance of exercise to the individual and tried not to hinder.

(Dylan, Focus group): But they [teammates] don't tell me not to exercise, just to 'take it easy'. And don't go all out full blow, as I like to when I'm playing. ... They won't tell me not to exercise because they know I enjoy it and they know it's good. But they've told me to take it easy and if I do feel tired, just sit out and stuff like that. They are pretty sensible with it. They won't hinder me from participating.

This support has also helped in boosting the confidence and motivation to exercise.

(Heather, Focus group) And my husband was really good at just going, 'Get out of bed, you can do it, you can do it.' I think it helps as well, to have that person help you with the exercise, cause then you sort of believe in yourself a bit more, if someone else does.

One way of providing this support is to create a safe haven in case a seizure occurs.

(Penelope, Focus group): My husband rides a bike. So I did quite fancy getting a bike and riding with him. But if I did, because I've never ridden a bike, never had one as a child, perhaps that's why, I'd want to ride behind him, not in front of him in all that traffic. But he said, 'If you're riding a bike, you're in front of me, so if I see you start wobbling I know something's going on.’

This being said, this same participant also reported a negative experience as a result of a lack

of knowledge of epilepsy by those within an exercise environment.

(Penelope, Focus group): We did go running with a particular group in Portsmouth and one time my husband couldn't go for a particular reason, and a couple of friends that usually go weren't there so I said to the guy who ran that particular group, 'Next time, I'll be coming by myself, is that ok?' 'No I'd rather you didn't.' I mean other people knew me and knew I had epilepsy but he said, 'I'd rather you didn't come without your friends or your husband.' 
374 As shown above, there was a mixture of responses in regard to social support and exercising

375 with epilepsy. This insight in regard to the positive and negative impact of social support on

376 the person with epilepsy maintaining exercise or taking part in team sports is important to

377 document.

\subsection{Discussion}

379

The barriers and adaptations used by PWE to exercise confirm previous literature as well as provided new insights into the methods used to overcome such barriers. Barriers to exercise, e.g., exercise type and intensity, as well as advice in regard to stopping exercise, have previously been shown $[1,4,5,6,11,12,14,19]$. However, to the best of our knowledge, overheating with exercise as a seizure trigger is a new addition to current barriers. Adding to these findings, insight into how a person with epilepsy adapts to such barriers through the use of technology or exercising at cooler times of the day is another key addition to current literature.

The participants' reports of enjoyment of exercise and feeling the benefits on their health status as a main motivator to exercise have been shown recently $[1,4,5]$. The positive impact on mood was discussed by the participants and was one motivator to continue to exercise. More detailed findings regarding mood improvement associated with exercise for PWE noted in this study will be presented in a future paper. These findings also revealed that participants felt exercise positively improved their health and allowed them to recover faster from a seizure. Exercise improving recovery from a seizure is, to the best of our knowledge, new to literature and important to explore further. A key psychological motivation consistent with the literature is 'not letting epilepsy stop me.' This confirms a common sentiment for PWE in relation to exercise as well as in everyday life $[4,5,10,19,20]$. This motivational strategy is important to note as it shows that PWE feel that through exercising, despite having 
uncontrolled seizures, they are gaining some control of their health. As epilepsy can often make PWE feel out of control of their health $[4,5,19,20]$, these findings show that it may be through a consistent exercise routine that PWE may be able to find an aspect of this control.

Although exercise-induced seizures are reported to be prevalent in only 1-2\% of PWE (21, 22), some of our participants reported exercise to be a trigger for their epilepsy. This was in connection with higher intensity exercise and overheating. The adaptations used and developed by the participants revealed that although certain aspects of exercise may trigger seizures for some, they felt continuing to exercise with adaptations was more beneficial to their overall mental and physical health.

In terms of aiding their exercise routine, participants discussed methods of adaptation and coping with the disruption of uncontrolled seizures. As safety was a main issue for many participants, it was through the processes of making sure to eat prior to exercise, exercising at a cooler time of day, as well as exercising with another person, that made the participants feel safer. Although not everyone reported the same adaptation techniques, the common thread throughout was that having epilepsy caused the participants to be wary of potential triggers and they needed to prepare themselves against them. For example, if overheating was a trigger, they would not exercise at the hottest point of day. Also, if he/she was feeling tired, he/she would rest that day to be able to exercise the next. Such self-management techniques have been developed as a result of finding what enables the most consistent exercise routine.

For the participants, one of the main methods of reducing the occurrence of a seizure was to adapt the exercise intensity level and type. For example, walking instead of playing cricket or playing in goal when playing soccer. This did not leave the participants with a feeling that their exercise level was sufficient. However, it did allow them to feel safe and able to continue to exercise. A recent report by the ILAE Task Force on Sports and Epilepsy 
422 shows the recommended exercise and sports for seizure type/control [13]. However, our

423

424 results show that although they feel safer, that does not always mean they are pleased with having to adapt their sport and/or exercise routine. The emotional impact of having to adapt their exercise intensity and frequency as a result of uncontrolled seizures confirms previous research $[4,5]$. Therefore, there remains a need to develop strategies to aid PWE to psychologically cope with this change in their life.

Another method of adaptation was through the use of technology. The reports of using heart rate monitors and temperature gauges to help decrease seizure triggers whilst exercising is a new addition to research. This adaptation method aided their exercise routine as it produced tangible data that they could relate to and use in self-management of their seizure frequency. As technology evolves and becomes easier to access, this may be one useful tool that will aid exercise adherence and consistency for PWE and is of importance to investigate in further studies.

Finally, social support was a key motivator whereas lack of social support was a key barrier in regard to exercise. Taking part in exercise with teammates or friends and family continues to allow PWE to feel safer whilst exercising. Recent research shows that exercising in a group or with another individual does increase the feelings of safety for PWE [5]. In addition, research has shown that partaking in exercise and sports can decrease seizure frequency and increase mental health [1, 2, 5, 13]. Though standard medical care from primary care physicians and neurologists is to maximize exercise and sports within the context of safety $[1,12,13,19]$, medical advice to limit exercise has been noted in previous research and, as seen within our findings, can be a potential barrier preventing PWE to exercise confidently and safely $[4,5,19,23]$. Since the advice to participate or not in exercise differed across the participants, further research needs to examine medical professionals’ knowledge of current exercise advice for PWE and if such advice is being offered to their 
447 patients. Educating medical professionals on best practices for PWE in regard to exercise and

448 sports may be a key avenue to pursue to increase physical activity levels [1, 3, 13]

\section{$449 \quad 4.1$ Limitations}

As a small $(\mathrm{N}=11)$ exploratory study, findings presented relate to the participants included and cannot be generalized for all PWE. However, this study has raised important issues which may be of relevance to other PWE and has provided new avenues for further research. Furthermore, the demographics of the participants need to be taken into consideration, as there was a lack of diversity, majority of females to males, and absence of socioeconomic details. In addition, as the researcher herself had epilepsy, this can be seen as a positive as well as a possible hindrance to the research. However, any negative influences that could have impacted the research were avoided as a result of reflection (discussed in section 2.4) and discussion of topics and analysis with the co-researcher.

\subsection{Conclusion}

This research has provided a first-person perspective on current barriers to exercise and adaptation methods for PWE. Presenting these new findings will allow for further consideration of how we may encourage more PWE to exercise. These findings show that exercise is important for PWE and self-management techniques are being developed as a result of their own desire to maintain a healthy body and mental state. This exploratory study suggests that mixed exercise advice for PWE persists. Further research (more participants with increased PWE diversity) is needed to examine current exercise advice offered to PWE from healthcare professionals (primary care physicians, specialists, nurses, etc.). This research could lead to educating more medical professionals about the benefits of exercise for PWE as well as what the current guidelines advise. Providing PWE with a voice within 
research has allowed for further acknowledgement that exercise is beneficial and that

adaptation and benefits need to be explored further in order to provide a higher quality of life.

\section{Acknowledgements}

We would like to thank all the participants who gave their time and spoke openly with us. We appreciate all they have done to further the research on exercising with epilepsy.

\section{Conflict of interest}

The authors declare no conflict of interest.

\subsection{References}

1. Pimental J, Tojal R, Morgado J. Epilepsy and physical exercise. Seizure 2015; 25: 87-94.

2. Arida RM, Guimaraes de Almeida AC, Cavalheiro EA, Scorza FA. Experimental and clinical findings from physical exercise as complementary therapy for epilepsy. Epilepsy Behav 2013; 26: 273-78.

3. McAuley JW, Long L, Heise J, Kirby T, Buckworth J, Pitt C, et al. A prospective evaluation of the effects of a 12-week outpatient exercise program on clinical and behavioral outcomes in patients with epilepsy. Epilepsy Behav 2001; 2: 592-600.

4. Scarfe SV, Marlow C. Overcoming the fear: an autoethnographic narrative of running with epilepsy. Qual Res Sport Exercise Health 2015;7(5):688-97.

5. Collard SS, Marlow C. The psychosocial impact of exercising with epilepsy: A narrative analysis. Epilepsy Behav 2016; 61: 199-205.

6. De Lima C, de Lira CAB, Arida, RM, Andersen ML, Matos G, de Figueiredo Ferreira Guilhoto LM et al. Association between leisure time, physical activity, and mood disorder levels in individuals with epilepsy. Epilepsy Behav 2013; 28: 47-51.

7. Cui W, Zack MM, Kobau R, Helmers SL. Health behaviors among people with epilepsyResults from the 2010 National Health Interview Survey. Epilepsy Behav 2015; 44: 121-26.

8. Steinhoff BJ, Neususs K, Thegeder H, Reimers, CD. Leisure time activity and physical fitness in patients with epilepsy. Epilepsia 1996; 37(12): 1221-27.

9. Arida RM, Scorza FA, de Alburquerque M, Cysneiros RM, de Oliveira RJ, Cavalheiro EA. Evaluation of physical exercise habits in Brazilian patients with epilepsy. Epilepsy Behav 2003; 4: 507-510. 
10. Kaufman KR, Kaufman ND. Stand up for epilepsy San Diego photo shoot: A personal odyssey. Epileptic Disord 2013;15(2):211-5

11. Arida RM, Cavalheiro EA, da Silva AC, Scorza FA. Physical activity and epilepsy. Sports Med 2008; 38(7): 607-15.

12. Sirven J I. Epilepsy and exercising: Another brick in the wall? Epilepsy Behav 2009; 14: $1-2$.

13. Capovilla G, Kaufman KR, Perucca E, Moshé SL, Arida RM. Epilepsy, seizures, physical exercise, and sports: A report from the ILAE task force on sports and epilepsy. Epilepsia 2016; 57(1):6-12.

14. van Teijlingen E, Pitchforth E. Focus group research in family planning and reproductive health care. J Fam Plann Reprod Health Care 2006;32 (1): 30-32.

15. Folch-Lyon E, Trost JF. Conducting focus group sessions. Studies in Family Planning 1981; 12 (12): 443-449.

16. Higginbottom G, Lauridsen EI. The roots and development of constructivist grounded theory. Nurse Res 2014; 21(5):8-13.

17. Charmaz K. Constructing Grounded Theory. London: Sage Publications; 2006.

18. Creswell JW. Qualitative inquiry and research design: Choosing among five approaches. $3^{\text {rd }}$ edition. London: Sage Publications; 2013.

19. Ablah E, Haug A, Konda K, Tinius AM, Ram S, Sadler T, et al. Exercise and epilepsy: A survey of Midwestern epilepsy patients. Epilepsy Behav 2009; 14: 162-166.

20. Jacoby A. Stigma, epilepsy, and quality of life. Epilepsy Behav 2002; 3: S10-S20.

21. Kamel JT, Badawy RAB, Cook MJ. Exercise-induced seizures and lateral asymmetry in patients with temporal lobe epilepsy. Epilepsy Behav Case Rep 2014; 2: 26-30.

22. Nakken KO, Physical exercise in outpatients with epilepsy. Epilepsia 1999; 40(5):643-51.

23. Sturm JW, Fedi M, Berkovic SF, Reutens DC. Exercise-induced temporal lobe epilepsy. Neurology 2002; 59(8): 1246-48. 
534

535

536

537

538

539

540

541 